\title{
Magnitude of $G_{2}$ reward and the frustration effect in a between-subjects design
}

\author{
R. A. KRIPPNER ${ }^{1}$, UNIVERSITY OF SOUTH DAKOTA
}

\author{
R. C. ENDSLEY, UNIVERSITY OF GEORGIA \\ R. S. TACKER, UNIVERSITY OF SOUTH DAKOTA
}

An investigation failed to indicate that differential second task reward magnitude was a salient determinant of the frustration effect (FE). However, the influence of variations of second task reward magnitudes on second task speed measures was observed.

Numerous studies generated by Amsel's theory of frustrative nonreward (1962) have indicated that after several trials of receiving reward for running to the goal boxes $\left(G_{1}, G_{2}\right)$ of two consecutive alleys ( $A_{1}$, $A_{2}$ ), rats run faster in $A_{2}$ following $G_{1}$ nonreward than following $G_{1}$ reward. This increase in speed following $G_{1}$ nonreward has been termed the "frustration effect" (FE). Generally, studies have concentrated on manipulating the incentive conditions in $\mathrm{G}_{1}$ that subsequently determine the strength of $\mathrm{FE}$. However, the possible role that $G_{2}$ incentive conditions may have on demonstrations of FE has been ignored. Thus, the purpose of the present study was to explore whether variations in $G_{2}$ reward magnitude affect FE.

At least three theoretical notions can be offered for the possible effects of $G_{2}$ reward magnitude on FE: (1) According to a "celling effect" hypothesis, demonstrations of $\mathrm{FE}$ might be more difficult if $\mathrm{G}_{2}$ incentives are increased. Large $G_{2}$ incentives might produce sufficently high $A_{2}$ running speeds such that further increments in speed following $G_{1}$ nonreward would not be possible.

(2) According to a "competing approach tendencies" hypothesis, demonstrations of FE might be more difficult if the magnitude of $\mathrm{G}_{1}$ reward is small relative to $G_{2}$ rewards. It can be assumed that variations in the relative magnitudes of reward presented in $G_{1}$ and $G_{2}$ alter the strengths of competing approachapproach tendencies toward $G_{1}$ and $G_{2}$. The findings of McHose \& Ludvigson (1965), and trends in the data of Peckham \& Amsel (1964) and Krippner, Endsley, \& Tacker (1967) that $A_{2}$ speed measures are depressed by increasing amounts of $G_{1}$ rewards, could be interpreted as reflecting stronger tendencies of Ss under large $G_{1}$ reward to return to or stay in $G_{1}$ instead of approaching $G_{2}$ (Krippner et al, 1967). A competing tendencies hypothesis could also help explain the fallure of McHose and Ludvigson, and Barrett, Peyser, \& McHose (1965) to demonstrate FE following incomplete $G_{1}$ reward reduction. Briefly, it is possible that $\mathrm{Ss}$ in these two studies did not show the $F E$ following nonzero reduction in $G_{1}$ as compared with control group $\mathrm{Ss}$ because of stronger competing tendencies in the former Ss to remain in or return to $G_{1}$. This result would be expected since the Ss receiving reward reduction were accustomed to relatively larger $G_{1}$ rewards than $G_{2}$ rewards in comparison with control $\mathrm{Ss}$.

(3) According to an "incentive-summation" hypothesis, demonstrations of FE might be enhanced as the size of $G_{2}$ reward is increased. According to the characteristics customarily accorded the hypothetical anticipatory goal response $\left(r_{r}\right)$ and its attendant stimulus $\left(s_{r}\right)$, the anticipatory goal responses elicited in $G_{2}\left(r_{r 2}\right)$ should eventually be aroused in the beginning portions of $A_{2}$. If it can be further assumed that $R_{r 1^{-S}}$ elicited in $G_{1}$ carry over into $A_{2}$ (Bower, 1962), then conditioning of $\mathrm{S}_{\mathrm{r} 1}-\mathrm{r}_{\mathrm{r} 2}$ would be expected. Granted these assumptions, $A_{1}$ would eventually come to elicit both $r_{r 1}$ and $r_{r 2}\left(A_{1}-r_{r 1}{ }^{-S} r_{r 1}-r_{r 2}\right)$. Therefore, it would be predicted that increasing $\mathrm{G}_{2}$ reward magnitude would amplify $F E$ because of summated approach tendencies present in $A_{1}$ preceding $G_{1}$ nonreward.

\section{Method}

The Ss were 18 experimentally naive, male LongEvans hooded rats ranging in age from 100-110 days at the start of the experiment. The apparatus was an L-shaped double runway with a startbox $\left(S_{1}\right)$, alley $\left(A_{1}\right)$, and goalbox $\left(G_{1}\right)$ comprising the first runway and an alley $\left(A_{2}\right)$ and goalbox $\left(G_{2}\right)$ comprising the second runway.

At the start of the experiment, the 18 Ss were randomly assigned to three groups, 5-1, 5-5, and 5-10. The group numbers represented the respective number of $45 \mathrm{mg}$ Noyes pellets $\mathrm{Ss}$ would receive in $G_{1}$ and in $G_{2}$ on any rewarded trial. After 10 days of handling and familiarization with the apparatus, Ss were given 72 acquisition trials followed by $\mathbf{7 2}$ test trials. The Ss were given four trials a day during acquisition and testing phases. On each trial during acquisition and testing, measures of start time and running time were taken in $A_{2}$. The procedure for test trials was identical with that on acquisition trials except that nonreward was introduced in $G_{1}$ on half of the test trials. During each block of four test trials, all groups received $G_{1}$ reward (R) or nonreward (N) trials according to the following repeating sequence: RNRN/RNNR/NRNR/NRRN.

Additional details regarding the apparatus, maintenance schedule, and pretraining, acquisition, and testing procedures may be found in Krippner et al (1967). 

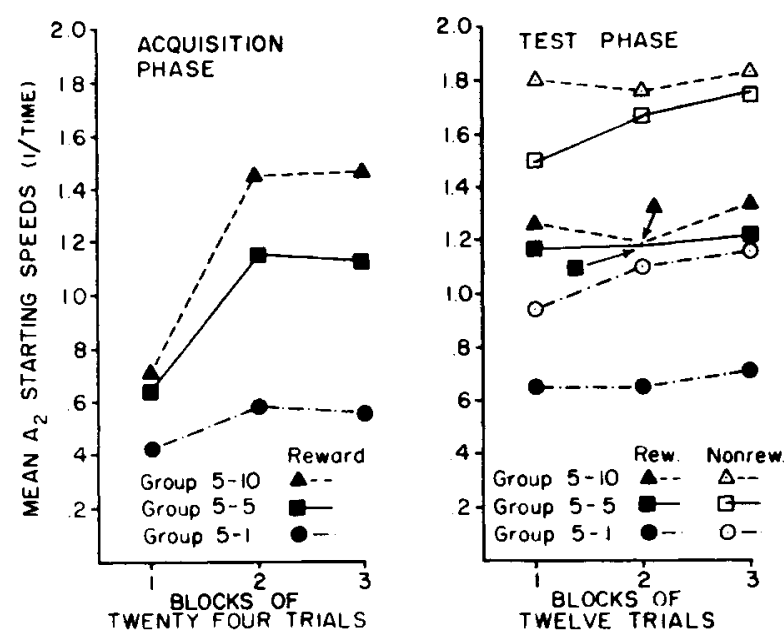

Fig. 1. Mean $A_{2}$ starting speeds as a function of trial blocks.

\section{Results and Discussion}

The start and running time data were reciprocalized and treated as start and running speeds. Performance of the start speeds of Groups 5-1, 5-5, and 5-10 is shown in Fig. 1.

A two factor analysis of variance with repeated measures on one factor was performed on the acquisition start speed data. The analysis yielded a significant Groups effect $(F=8.8, \mathrm{df}=2 / 15, p<.01)$, Trial Blocks effect $(F=28.7, d f=2 / 30, p<.01)$, and Groups by Trials interaction $(F=4.21, d f=4 / 30, p<.01)$. Inspection of Fig. 1 indicates that the Groups effect was due to a positive relationship between amount of $G_{2}$ reward and $A_{2}$ starting speed, while the Groups by Trials interaction was reflected in increasing differences among Groups 5-10, 5-5, and 5-1 over successive trials. The increasing divergence among groups over trials reflected the positive relationship between running speed and magnitude of reward.

A three factor analysis of variance with repeated measures on two factors was performed on the test trial starting speeds, with Groups, Reward Conditions (reward-nonreward) and Trial Blocks as the main effects. Just preceding the test trials, one $S$ from Group 5-5 died due to a respiratory illness. Therefore, the mean raw score for each trial of the five remaining $\mathrm{Ss}$ in Group 5-5 was assigned to the deceased $\mathrm{S}$, and one degree of freedom was subtracted from the total and from the error sums of squares in order to correct for the missing data. The analysis yielded significant effects of Trial Blocks $(F=4.1, d f=2 / 28, p<.05)$. Reward Conditions $(F=$ 190.9, df $=1 / 14, p<.001)$, and the Reward Conditions by Trial Blocks interaction $(F=4.5, d f=2 / 28, p<.05)$. None of the other effects was significant.

Inspection of Fig. 1 indicated that the significant Reward Conditions effect reflected the appearance of $\mathrm{FE}$ in each group. However, as reflected in the nonsignificant Reward Conditions by Groups interaction, there is no evidence in Fig. 1 that variations in $G_{2}$ magnitude differentially affected the demonstration of $\mathrm{FE}$.

Analysis of variance of the acquisition and test running speed data produced significant effects identical to those found in the start speed analysis with one exception. The Groups by Trials interaction was not significant in the acquisition running data.

On the basis of the present results it must be concluded that while increases in $G_{2}$ reward magnitudes generally increase start and running speeds both following $G_{1}$ reward and nonreward, there is no evidence to support any of the hypotheses regarding the role of $G_{2}$ reward magnitude on demonstrations of FE.

\section{References}

AMSEL, A. Frustrative nonreward in partial reinforcement and discrimination learning: Some recent history and a theoretical extension Psychol Rev., 1962, 69, 306-328.

BARRETT, R. J., PEYSER, C. S., \& McHOSE, J. H. Effects of complete and incomplete reward reduction on a subsequent response. Psychon. Sci, 1965, 3, 277-278.

BOWER, G. H. The influence of graded reduction in reward and prior frustrating events upon the magnitude of the frustration effect. $J$. comp. physiol Psychol, 1962, 55, 582-587.

KRIPPNER, R. A., ENDSLEY, R. C., \& TACKER, R. S. Magnitude of $G_{1}$ reward and the frustration effect in a between-subjects design. Psychon. Sci, 1967, 9, 385.386.

McHOSE, J. H., \& LUDVIGSON, H. W. Role of reward magnitude and incomplete reduction of reward magnitude in the frustration effect. J. exp. Psychol, 1965, 70, 490-495.

PECKHAM, R. H. JR., \& AMSEL, A. Magnitude of reward and the frustration effect in a within-subjects design. Psychon Sci, 1964, 1, 285286.

Note

1. This investigation was carried out during the tenure of a National Defense Education Act Title IV Pre-doctoral Fellowship. 